\title{
A multi-parameter calibration method for the numerical simulation of morphodynamic problems
}

\author{
Stefania Evangelista ${ }^{1 *}$, Gaspare Giovinco $^{1}$, Selahattin Kocaman ${ }^{2}$ \\ ${ }^{1}$ Department of Civil and Mechanical Engineering DiCeM, University of Cassino and Southern Lazio, 03043 Cassino, FR, Italy. \\ ${ }^{2}$ Department of Civil Engineering Technical University of Iskenderun 31200 Iskenderun, Hatay, Turkey. \\ ${ }^{*}$ Corresponding author. E-mail: s.evangelista@unicas.it
}

\begin{abstract}
Calibration of parameters of mathematical models is still a tough task in several engineering problems. Many of the models adopted for the numerical simulations of real phenomena, in fact, are of empirical derivation. Therefore, they include parameters which have to be calibrated in order to correctly reproduce the physical evidence. Thus, the success of a numerical model application depends on the quality of the performed calibration, which can be of great complexity, especially if the number of parameters is higher than one. Calibration is traditionally performed by engineers and researchers through manual trial-and-error procedures. However, since models themselves are increasingly sophisticated, it seems more proper to look at more advanced calibration procedures. In this work, in particular, an optimization technique for a multi-parameter calibration is applied to a two-phase depth-averaged model, already adopted in previous works to simulate morphodynamic processes, such as, for example, the dike erosion by overtopping.
\end{abstract}

Keywords: Optimization; Calibration; Multi-parameter; Numerical models; Morphodynamic models; Dike erosion.

\section{INTRODUCTION}

The calibration of parameters of the physically-based mathematical models governing different problems is still a difficult task in engineering.

Many of the models adopted for the numerical simulations of real phenomena, in fact, are of empirical derivation. As a consequence, they often include coefficients that cannot be measured directly due to measurement limits and scales issues and, therefore, have to be calibrated in order to correctly reproduce the physical evidence. Thus, the success of the application of any numerical model is strongly dependent on how precisely the model is calibrated.

However, for most models the calibration of the parameters can be of great complexity, especially for large number of model parameters.

Engineers and researchers traditionally count on knowledge and experience with the model to adjust the parameters through manual trial-and-error procedures and comparison against available experimental data, often varying a single parameter and assuming the other ones as constant. Yet, this approach to calibration is subjective and cumbersome. Since the models themselves are increasingly sophisticated, it seems more proper to look at more advanced calibration procedures. Modern hardware and software systems nowadays allow for high speed and capacity calculation, but the choice of an optimal method to effectively identify the set of parameter sets is still of some concern. Automatic calibration methods, at the same time objective and easy to implement with high speed computers, have become popular in recent years (e.g. Finley et al., 1998; Kleidorfer et al., 2009). Global optimization algorithms, for example, can efficiently search the set of parameters able to minimize (or maximize) objective functions quantifying the agreement between observations and simulations. On the other hand, a considerable amount of research has recently concerned modeling of fast geomorphic processes, and more complex morphodynamic multi-layer (e.g. Capart and Young, 2002; Li et al., 2013; Savary and Zech, 2007; Swartenbroekx et al., 2010) or multi-phase (e.g. Evangelista et al., 2013a; Garegnani et al., 2011; Greco et al., 2012; Rosatti and Begnudelli, 2013) models have been proposed, which account for the mass and momentum conservation for both water and sediments (Evangelista et al., 2015c). The increased complexity of the morphodynamic models is rewarded by the possibility to better reproduce fast geomorphic transients. Most of these models include more than one empirical coefficients to be calibrated.

Nevertheless, the calibration is still entrusted in general to manual trial-and-error procedures. Only recently few attempts to make use of more sophisticated automatic approaches for the calibration of morphodynamic models can be found in the literature, but especially in the fields of Hydrology and Coastal Engineering (e.g. Lo et al., 2015; Smith et al., 2009, 2013), rather than in Fluvial Hydraulics.

In this work a global optimization algorithm, and, specifically, the derivative free Nelder-Mead algorithm (Nelder and Mead, 1965) has been adopted to optimize the multi-parameter calibration of a two-phase depth-averaged model (Greco et al., 2012), already applied in previous works to simulate morphodynamic processes, such as, for example, the dike erosion by overtopping (Evangelista, 2015; Evangelista et al., 2015a).

Results are encouraging and suggest that the optimization algorithm here proposed can be possibly successfully applied to different mathematical models in river hydraulics and in general in the fields of hydraulics involving geomorphic processes (erosion/deposition of movable bottoms, sediment transport).

\section{DIKE EROSION BY WAVE OVERTOPPING}

The optimization procedure is applied here to the numerical simulation of some small-scale experiments of dike erosion by wave overtopping conducted in the Water Engineering Laboratory (LIA Lab) at University of Cassino and Southern Lazio, Cassino (FR), Italy. These experiments have been performed in a horizontal rectangular channel (Evangelista et al., 2015a; Evangelista et al., 2014b), where a dam break is simulated through the sudden opening of a gate closing an upstream reservoir (Fig. 1). A downstream trapezoidal sand dike is built upon a wooden ramp and, therefore, the dike overtopping occurs 


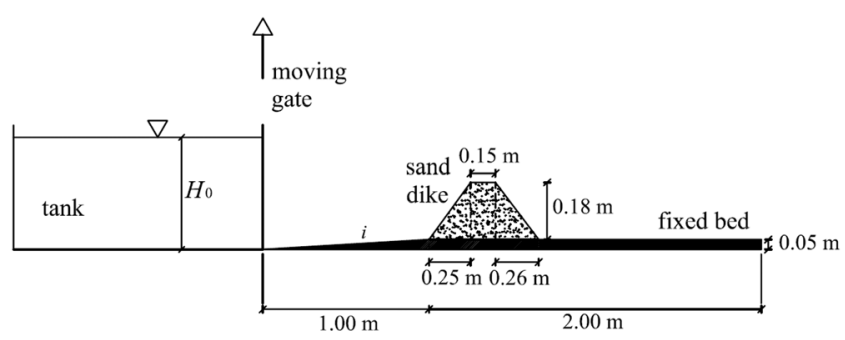

Fig. 1. Sketch of the laboratory physical model (lateral view).

if the hydrodynamic energy possessed by the wave is sufficient (Evangelista, 2015).

Different tests have been performed starting from different initial water levels in the reservoir and using two different almost-uniform non-cohesive sands (Evangelista et al., 2014a) for the dike structure. Specifically, three initial configurations, illustrated for both sands in Table 1 and corresponding to the three initial water levels $H_{0}$, have been considered. The dike height $H_{d}$ is the same for all tests and equal to $0.179 \mathrm{~m}$.

In the first scenario $\left(H_{0}=0.20 \mathrm{~m}\right.$, Tests $\mathrm{A} 1$ and $\left.\mathrm{B} 1\right)$, the initial water level in the upstream reservoir is almost equal to the dike top elevation. The dam break wave originating from the gate opening, therefore, does not possess enough energy to overtop the dike and induces erosion only on the upstream slope of the dike.

In the second case $\left(H_{0}=0.25 \mathrm{~m}\right.$, Tests $\mathrm{A} 2$ and B2), the initial upstream level is such that the water wave overtops the dike and erodes it along its entire profile.

In the third situation $\left(H_{0}=0.395 \mathrm{~m}\right.$, Tests $\mathrm{A} 3$ and $\left.\mathrm{B} 3\right)$, the potential energy of the impact wave is almost double that in the previous case, thus causing a more evident erosion of the dike.

Table 1. List of performed tests.

\begin{tabular}{llll}
\hline & \multicolumn{3}{c}{$\boldsymbol{H}_{\mathbf{0}}(\mathbf{c m})$} \\
Test & 20 & 25 & 39.5 \\
\hline Fine sand & A1 & A2 & A3 \\
Coarse sand & B1 & B2 & B3 \\
\hline
\end{tabular}

A computer-controlled camera is positioned laterally with respect to the channel with transparent walls, in order to record (with an acquisition frame rate of $14.5 \mathrm{~Hz}$ ) the evolution of the water wave and of the dike erosion process (Fig. 2). The evolution of the dam-break wave and the closely-connected evolution of the dike profile are investigated through image-analysis measuring techniques.

Preliminary tests demonstrated, in fact, that the process may be considered approximately 1D. The longitudinal section detected from the images on the sidewall can be, thus, assumed as representative of any other longitudinal section in the flow. A regular grid is also attached to the channel walls in order to permit proper rectification and scaling of the recorded images and, consequently, the correct measurement operation.

For each frame, the water surface and dike profiles along the channel are, therefore, evaluated through pre-processing and edge-detection tool software, also useful for the fast and objective processing of a great number of images (Evangelista, 2013; Evangelista et al., 2013a). The adopted reference frame axes coincide with the channel bottom and the gate position in the longitudinal and vertical directions, respectively (Evangelista, 2015). In this way, the evolution of the time- and spacedependent processes can be properly evaluated.

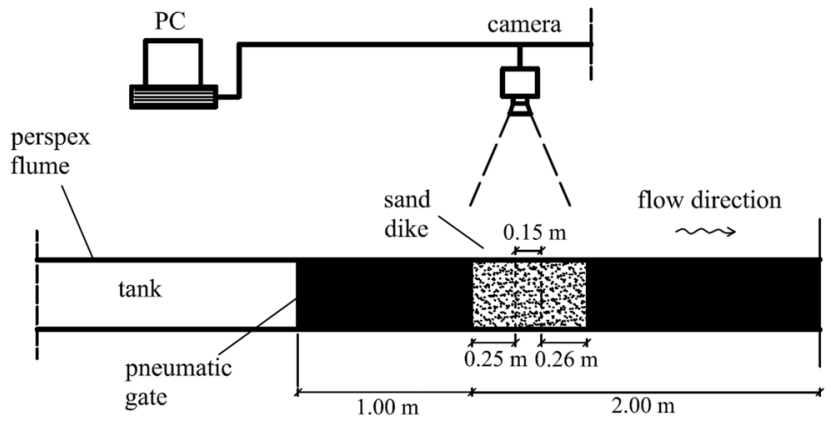

Fig. 2. Sketch of the laboratory physical model (top view).

The obtained experimental data have been used already in a previous work (Evangelista, 2015) to test the predictive capability of a numerical code based on a morphodynamic depthintegrated two-phase approach (Greco et al., 2012).

\section{NUMERICAL MODEL AND CALIBRATION PROCEDURE}

The model (Greco et al., 2012) adopted for the numerical simulation of the above described experiments (see also Evangelista et al., 2013a, b) neglects cohesion, suspended load sediment transport and seepage effects, but it is still capable of catching some features of the physical evidence, offering some indications for prediction and practical use.

The model is based on a depth-integrated two-phase approach, under the assumptions of the hydrostatic distribution of pressures, bed load sediment transport and a uniform noncohesive sediment, and includes a geo-failure operator (Evangelista et al., 2013b, 2015c). Conservation equations of mass and momentum are written for the water and solid phases separately, with a closure relation for the bed deformation rate.

The numerical integration of the model is performed using a mixed cell-centered and node-centered finite-volume discretization over unstructured triangular meshes and the first-order Harten-Lax-Van Leer (HLL) solver for the flux calculation (Harten et al., 1983), evolved to a second-order one for the water surface elevation (Evangelista et al., 2013a), with a specific wet-dry treatment (Greco et al., 2008). This model includes also a geo-failure operator that permits reproducing the failure mechanisms induced by the water rising over the steep slopes of river beds and banks, which significantly affect geomorphic flows. The operator is based on a limiting slope stability criterion: when in the cell the bed slope exceeds a critical angle, depending on the saturation degree of the sand, the bottom collapse occurs and the corresponding bed material and pore water become available to flow, thus following the twophase flow dynamics. The algorithm also permits the failure to propagate from the single cell to the entire domain, preserving both the continuity of the bottom surface and the mass conservation (Evangelista et al., 2013b, 2015c).

The results of the laboratory experimental campaign have been utilized already (Evangelista, 2015) to validate the capability of the numerical model of predicting the physical evidence and its reliability for analyzing also this kind of scenario.

In the previous work simulations have been run with a time step $\Delta t=0.001 \mathrm{~s}$ and a grid size $\Delta x=0.01 \mathrm{~m}$. Besides the sand physical parameters (density, mean diameter, porosity and friction angle), the parameters to be assigned in the model are the following: the Chezy coefficient $C h$, the friction coefficient expressing the shear exerted on the liquid phase while flowing over the erodible bed; the drag coefficient $C d$, involved in the 
momentum exchange between the liquid and the ensemble of bed-load sediment particles but lumps in it the particle shape factors; and finally the Bagnold coefficient $\alpha$ which regulates the amount of momentum loss by the sediment phase due to inter-particle collisions. Actually the latter was demonstrated to do not affect much the results (Evangelista et al., 2015c); this assertion was also proved, as explained in detail in section 4, with the optimization procedure of calibration.

The values for the Chezy $C h$ and drag $C d$ coefficients, instead, have been chosen, respectively, equal to 15 and 0.2 (see Evangelista et al., 2013a, 2015c) after a sensitivity analysis and a manual trial-and-error calibration procedure. A better calibration of the parameters has been sought in this work with a procedure aimed at optimizing the parameter calibration (see also Evangelista et al., 2015b).

In particular, in order to find the optimal values of the model coefficients (the $C h$ and $C d$ coefficients), the authors have solved the following minimization problem in Scilab (2012) through the Nelder-Mead algorithm (Nelder and Mead, 1965):

$$
\begin{gathered}
\min \left\{\sum _ { i = 1 } ^ { N } \left[\left(\left(\delta_{\text {num }, i}^{s}\left(\mathrm{C} h, \mathrm{C}_{d}\right)-\delta_{\text {exp }, i}^{s}\right)^{2}\right.\right.\right. \\
\left.\left.+\left(\delta_{\text {num }, i}^{w}\left(\mathrm{C} h, \mathrm{C}_{d}\right)-\delta_{\text {exp }, i}^{w}\right)^{2}\right]\right\}
\end{gathered}
$$

where $i$ is the considered time step, $\delta_{\text {num,i }}^{s}$ and $\delta_{\exp , i}^{s}$ are the numerical and experimental dike profiles for the $i$-th time step, respectively, and $\delta_{\text {num }, i}^{w}$ and $\delta_{\mathrm{exp}, i}^{w}$ are the numerical and experimental water surface profiles for the $i$-th time step, respectively.

The goal of the Nelder-Mead algorithm is is to solve the unconstrained optimization problem stated by:

$\min f(x)$

where $x \in \mathbb{R}^{n}, n$ is the number of optimization parameters and $f$ is the objective function $f: \mathbb{R}^{n} \rightarrow \mathbb{R}$.

The algorithm is based on the iterative update of a simplex made of $n+1$ points $S=\left\{\mathbf{v}_{i}\right\}_{i=1, n+1}$. Each point in the simplex, called vertex, is associated with a function value $f_{i}=f\left(\mathbf{v}_{i}\right)$, for $i=1, n+1$. All the vertexes are sorted by increasing function values so that the best vertex has index 1 and the worst vertex has index $n+1$, i.e.:

$$
f_{1} \leq f_{2} \leq \ldots \leq f_{n} \leq f_{n+1}
$$

The $\mathbf{v}_{1}$ and the $\mathbf{v}_{n+1}$ vertexes are called the best and the worst vertex respectively, since they are associated with the lowest and the highest function value, respectively.

Accordingly to the simplex vertexes, the centroid of the simplex $\overline{\mathbf{v}}(j)$ is the center of the vertexes where the vertex $v j$ has been excluded. This centroid is:

$$
\overline{\mathbf{v}}(j)=\frac{1}{n} \sum_{i=1, n+1, i \neq j} \mathbf{v}_{i}
$$

The algorithm attempts to replace some vertex $\mathbf{v} j$ by a new vertex $\mathbf{v}(\rho, j)$ on the line from the vertex $\mathbf{v} j$ to the centroid $\overline{\mathbf{v}}(j)$, by means of the so called reflection factor $\rho$, usually equal to 1:

$$
\mathbf{v}_{\mathrm{r}}=\mathbf{v}(\rho, j)=(1+\rho) \cdot \overline{\mathbf{v}}(j)-\rho \cdot \mathbf{v}_{j} .
$$

More in detail, the Nelder-Mead algorithm uses four parameters: the coefficients of reflection $\rho$, expansion $\chi$, contraction $\gamma$ and shrinkage $\sigma$. When the expansion or contraction steps are performed, the shape of the simplex is changed.

Moreover, the four parameters should satisfy the following inequalities

$\rho>0, \quad \chi>1, \quad \chi>\rho, \quad 0<\gamma<1, \quad 0<\sigma<1$

The standard values for these coefficients are:

$\rho=1, \quad \chi=2, \quad \gamma=\frac{1}{2}, \quad \sigma=\frac{1}{2}$

At each iteration, when the worst vertex is replaced through reflection by a new one accordingly to:

$\mathbf{v}_{\mathrm{r}}=\mathbf{v}(\rho, n+1)=(1+\rho) \cdot \overline{\mathbf{v}}(n+1)-\rho \cdot \mathbf{v}_{n+1}$,

a new value $f_{\mathrm{r}}=f\left(\mathbf{v}_{\mathrm{r}}\right)$ of the function is obtained.

From this new value, several possibilities can be followed, in order to obtain a better vertex:

If $f_{\mathrm{r}}<f_{1}$, the reflected point $\mathbf{v}_{\mathrm{r}}$ reduces the function value. Therefore, the algorithm tries to expand the simplex in order to reduce the function value even more:

$\mathbf{v}_{\mathrm{e}}=\mathbf{v}(\rho \cdot \chi, n+1)=(1+\rho \cdot \chi) \cdot \overline{\mathbf{v}}(n+1)-\rho \cdot \chi \cdot \mathbf{v}_{n+1}$

and the new function value $f_{\mathrm{e}}=f\left(\mathbf{v}_{\mathrm{e}}\right)$ is computed. If the function value is improved (reduced), the worst vertex $\mathbf{v}_{j}$ is replaced in the simplex by $\mathbf{v}_{\mathrm{e}}$. Otherwise, the reflection point $\mathbf{v}_{\mathrm{r}}$ is accepted.

If $f_{1}<f_{\mathrm{r}}<f_{n}$, the worst vertex $\mathbf{v}_{n+1}$ is replaced in the simplex by $\mathbf{v}_{\mathrm{r}}$.

If $f_{n}<f_{\mathrm{r}}<f_{n+1}$, the point:

$\mathbf{v}_{\mathrm{c}}=\mathbf{v}(\rho \cdot \gamma, n+1)=(1+\rho \cdot \gamma) \cdot \overline{\mathbf{v}}(n+1)-\rho \cdot \gamma \cdot \mathbf{v}_{n+1}$

is considered and $\mathbf{v}_{\mathrm{c}}$ is accepted if it is better than $\mathbf{v}_{\mathrm{r}}$. Otherwise, a shrink is performed by moving all vertexes toward the best one $\mathbf{v}_{1}$.

In all the other cases the following point is considered:

$\mathbf{v}_{\mathrm{c}}=\mathbf{v}(-\gamma, n+1)=(1-\gamma) \cdot \overline{\mathbf{v}}(n+1)+\gamma \cdot \mathbf{v}_{n+1}$

in this application the optimization algorithm was first applied calibrating the three parameters $(C h, C d$ and $\alpha)$ and then calibrating only the two parameters $C h$ and $C d$. The starting simplex was: 


$$
\begin{gathered}
C h \\
C d
\end{gathered}\left[\begin{array}{cccc}
20 & 21 & 22 & 23 \\
0.05 & 0.10 & 0.15 & 0.20 \\
10^{-6} & 5 \cdot 10^{-6} & 10^{-5} & 5 \cdot 10^{-5}
\end{array}\right]
$$

for the three-parameters optimization, and

$$
\begin{gathered}
C h \\
C d
\end{gathered}\left[\begin{array}{ccc}
20 & 21 & 22 \\
0.05 & 0.10 & 0.15
\end{array}\right]
$$

for the two-parameters optimization, respectively.

Convergence was reached if the absolute variation for each parameter and for the function was lower than $10^{-8}$.

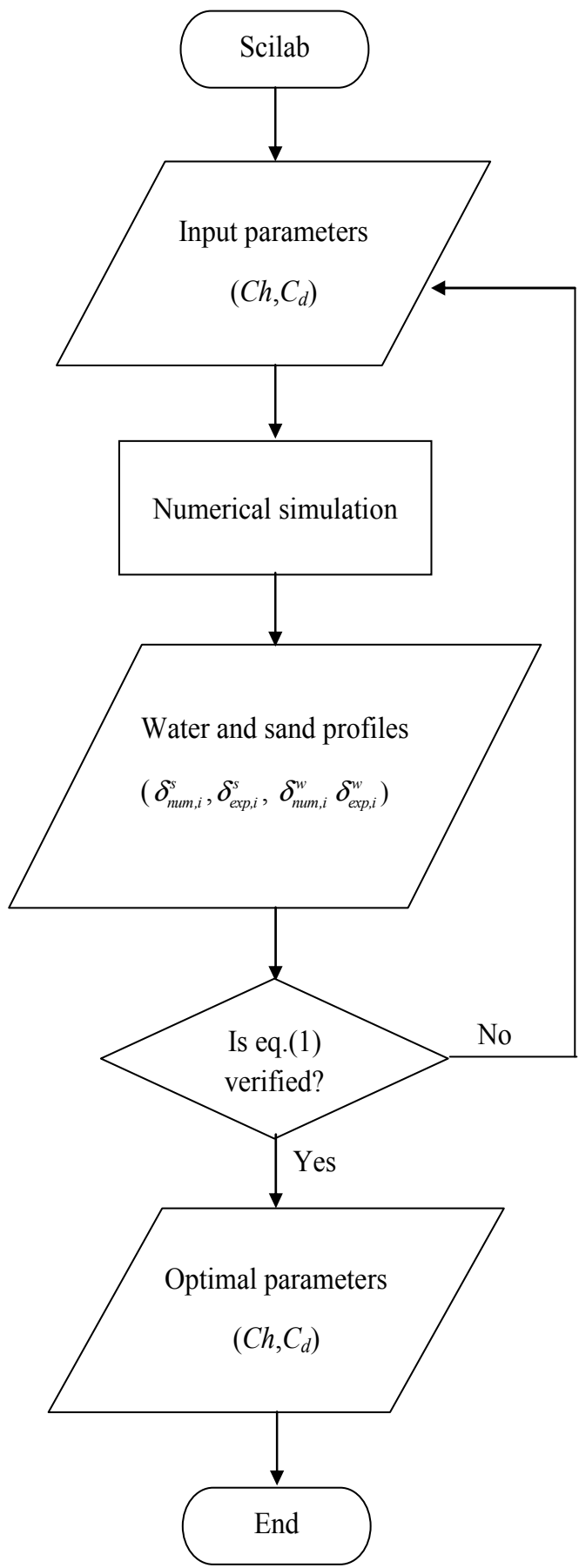

It has to be pointed out that a preliminary sensitivity analysis was made in order to determine the optimal number of time instants and the corresponding time values to be used in the optimization procedure. The maximum number of considered time instants was equal to 10 distributed in the range 0 to $15 \mathrm{~s}$. The analysis was performed taking into account also the computational efficiency.

The results show that the optimal number of time steps is three. An increase of the number of the considered time values led to a variation of the estimated parameters lower than $2 \%$, with, on the other side, a significant increase of the computational time. The selected times have been $1.40 \mathrm{~s}, 3.00 \mathrm{~s}$ and $15.00 \mathrm{~s}$. A shift of their position in the time scale did not lead as well to significant variations of the optimization results.

The flowchart of the optimization procedure is reported in Fig. 3.

The computational time required for each performed test was about 8 hours on a single $2.35 \mathrm{GHz}$ Intel ${ }^{\circledR}$ Core 2 Quad CPU computer with 8.00 GB RAM.

\section{RESULTS}

The optimization procedure gave also the chance to test the opportunity of effectively neglecting the influence of the Bagnold coefficient $\alpha$, as done in the previous work, which actually seems important in the model but whose variability was demonstrated to not affect much the results (Evangelista et al., 2015c). The three-parameter optimization results, obtained taking into account also the $\alpha$ coefficient and shown in Table 2, confirm that this assumption is acceptable.

Table 2. Results of the three-parameter calibration.

\begin{tabular}{|c|c|c|c|}
\hline TEST & $\boldsymbol{C h}$ & $\boldsymbol{C d}$ & $\boldsymbol{\alpha}$ \\
\hline A1 & 23.33 & 0.120 & $4.64 \mathrm{E}-05$ \\
\hline A2 & 23.47 & 0.101 & $1.07 \mathrm{E}-06$ \\
\hline A3 & 37.98 & 0.083 & $6.96 \mathrm{E}-05$ \\
\hline
\end{tabular}

The optimization algorithm was, therefore, applied to the calibration of only two parameters. Specifically, as mentioned before, three different times after the gate removal have been considered: $1.40 \mathrm{~s}, 3.00 \mathrm{~s}$ and $15.00 \mathrm{~s}$, respectively. The optimization has been performed with the aim of obtaining at the same time the best fit between both the water and the sand profiles at all the selected times, according to Equation (1). However, the algorithm is flexible enough to permit to set priorities in the optimization process, for example to seek for a better fit of the water profile with respect to the dike one, or to prefer a better correspondence at long instants after the gate removal respect to the initial ones. Results for all tests (A1-A3 and B1-B3) are summarized in Table 3.

Table 3. Results of the two-parameter calibration for all tests.

\begin{tabular}{|c|c|c|}
\hline TEST & $\boldsymbol{C h}$ & $\boldsymbol{C d}$ \\
\hline A1 & 24.82 & 0.110 \\
\hline A2 & 24.28 & 0.107 \\
\hline A3 & 32.53 & 0.074 \\
\hline B1 & 21.21 & 0.125 \\
\hline B2 & 24.80 & 0.115 \\
\hline B3 & 21.21 & 0.125 \\
\hline
\end{tabular}

Fig. 3. Flowchart describing the whole optimization procedure. 


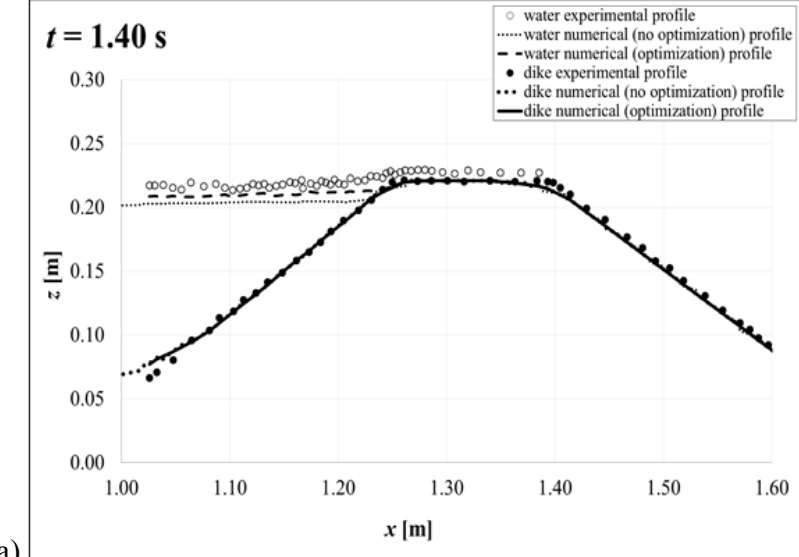

a)

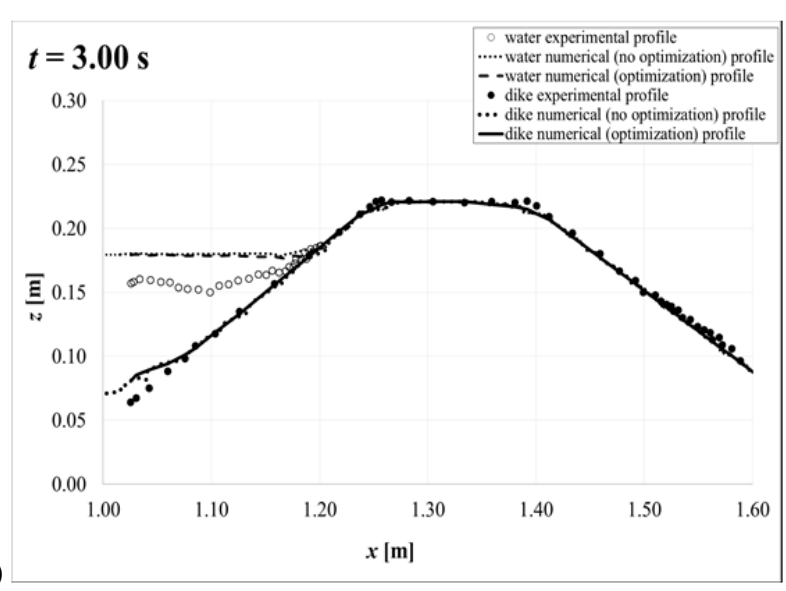

b)

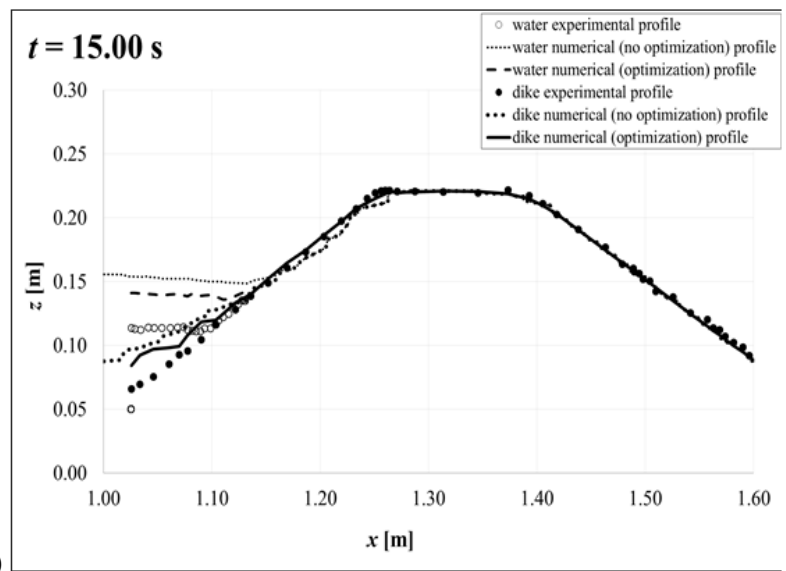

Fig. 4. Test A1: comparison between numerical (with and without optimization) and experimental results in terms of water and dike profiles at times a) 1.40 , b) 3.00 and c) $15.00 \mathrm{~s}$, respectively, after the gate removal.

Some comparisons of the experimental results against the numerical ones, respectively simulated in the previous work by Evangelista (2015) (in black) and with the optimization procedure (in grey) are illustrated in Figs. 4-6 for tests A and in Figs. 7-9 for tests B. Specifically, the water-surface and dike profiles at specific times, a) 1.40 , b) 3.00 and c) $15.00 \mathrm{~s}$, respectively, after the gate removal are plotted for tests A1, A2, A3, B1, B2, B3.

The observation of the comparisons in Figs. 4-9 proves a general improvement in the numerical results in terms of correspondence with the experimental data, respect to the ones obtained through the trial-and-error calibration performed in the previous work (Evangelista, 2015). A better agreement with the experimental curves is obtained on the average with the optimi-

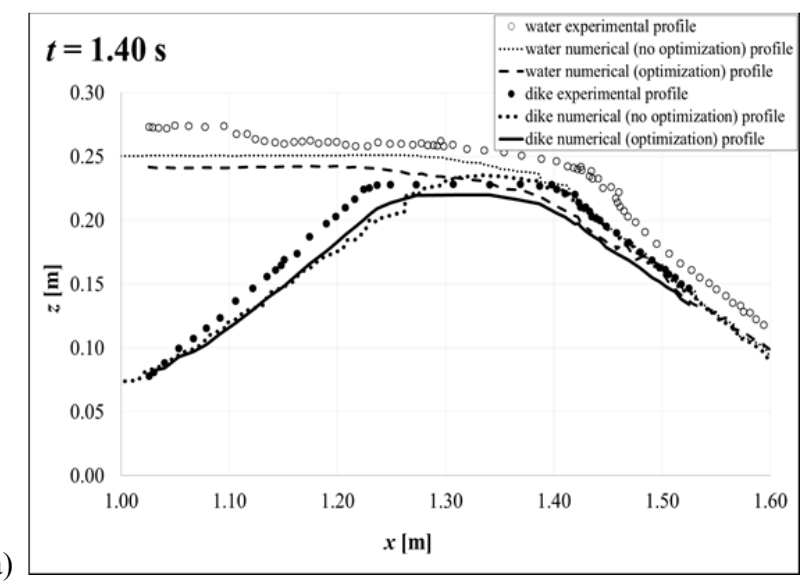

a)

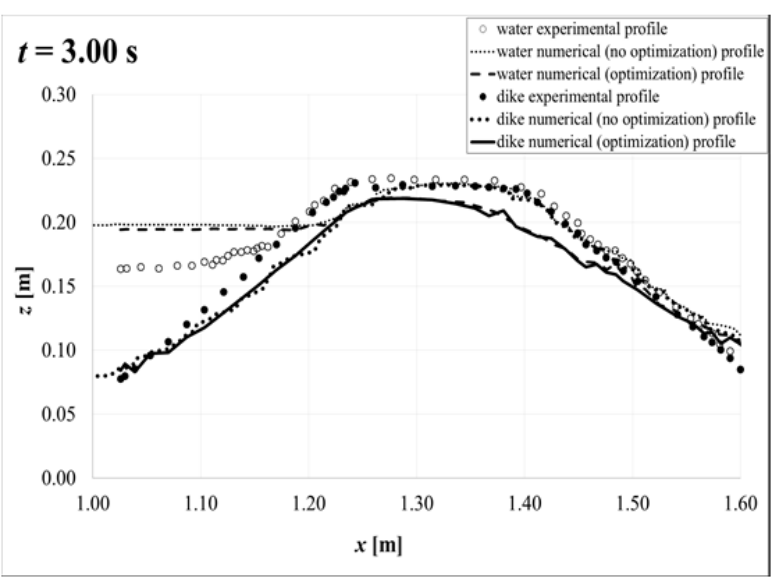

b)

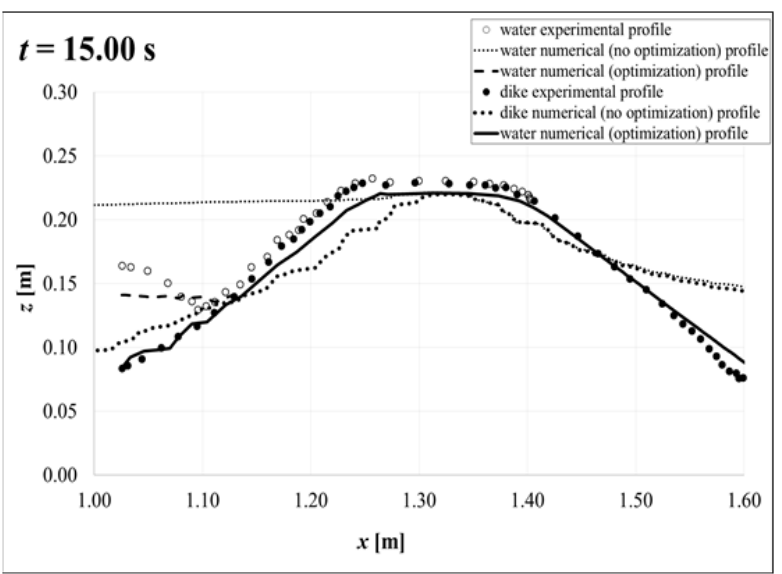

Fig. 5. Test A2: comparison between numerical (with and without optimization) and experimental results in terms of water and dike profiles at times a) 1.40, b) 3.00 and c) $15.00 \mathrm{~s}$, respectively, after the gate removal.

zation procedure application. In few cases the accuracy of numerical results achieved with and without optimization are comparable. However, the advantages of the calibration optimization are undeniable in terms of calculation times, computational efficiency and, least but not last, objectivity of the results, in comparison with a trial-and-error calibration. This traditional procedure, in fact, requires long processing times and laborious tasks, and it is certainly affected by the subjectivity of the operator and of the phases of the process.

In particular, the maximum relative differences between the numerical results and the experimental data are reported in Table 4, showing the best performance of the proposed methodology. 
a)

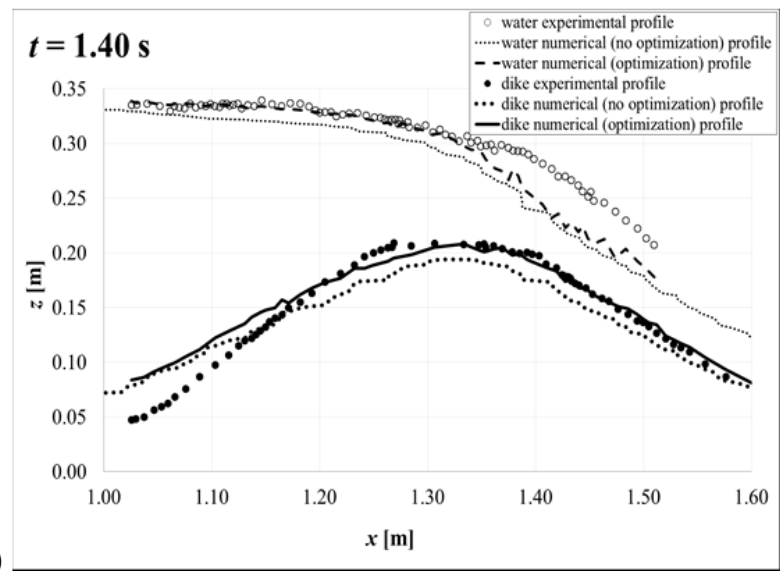

b)

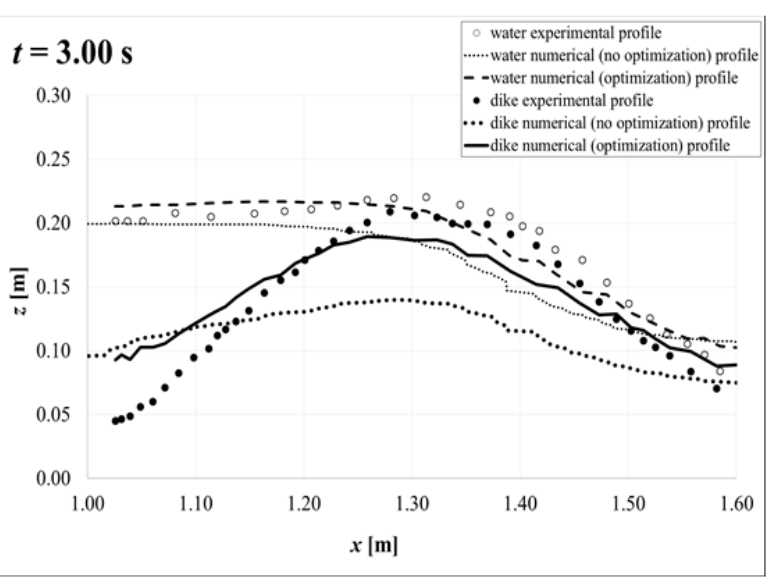

c)

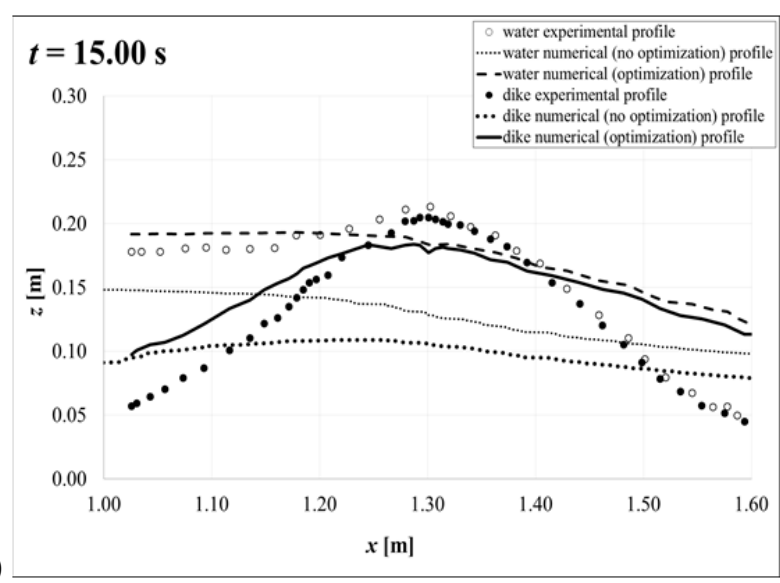

Fig. 6. Test A3: comparison between numerical (with and without optimization) and experimental results in terms of water and dike profiles at times a) 1.40, b) 3.00 and c) $15.00 \mathrm{~s}$, respectively, after the gate removal.
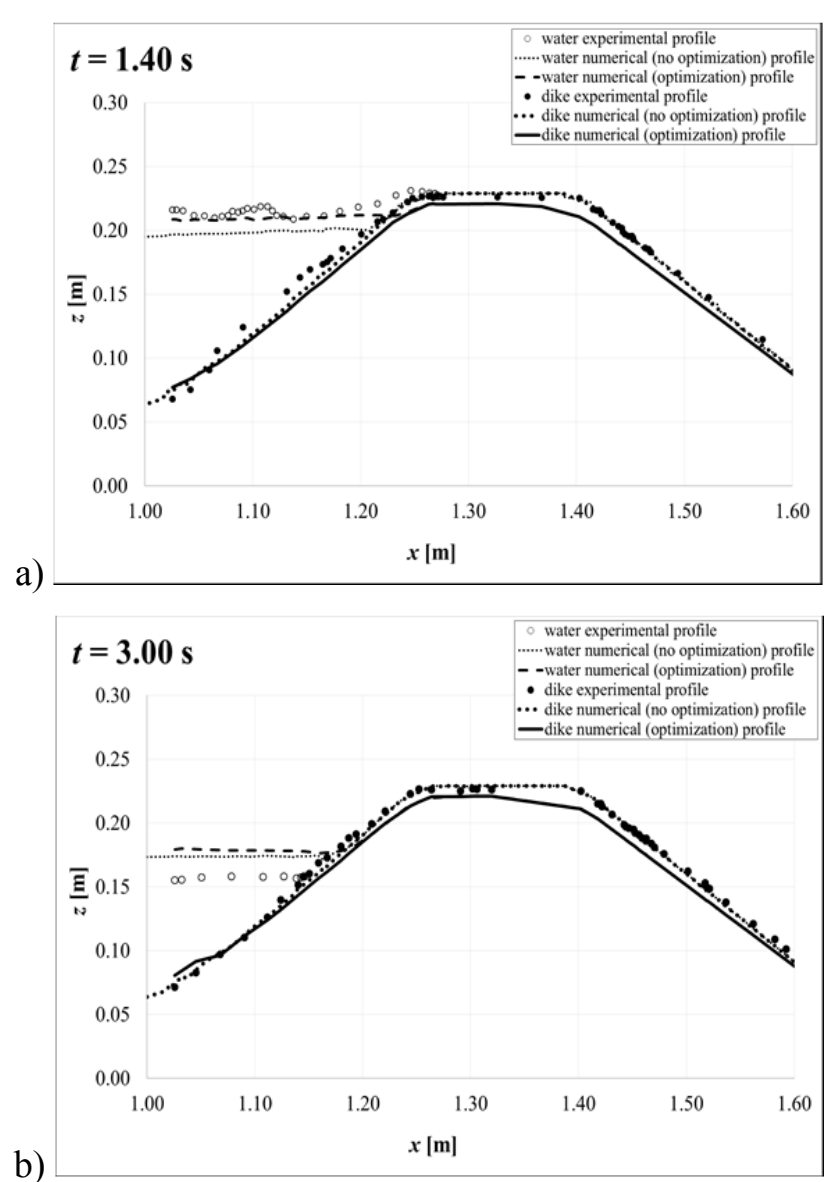

b)

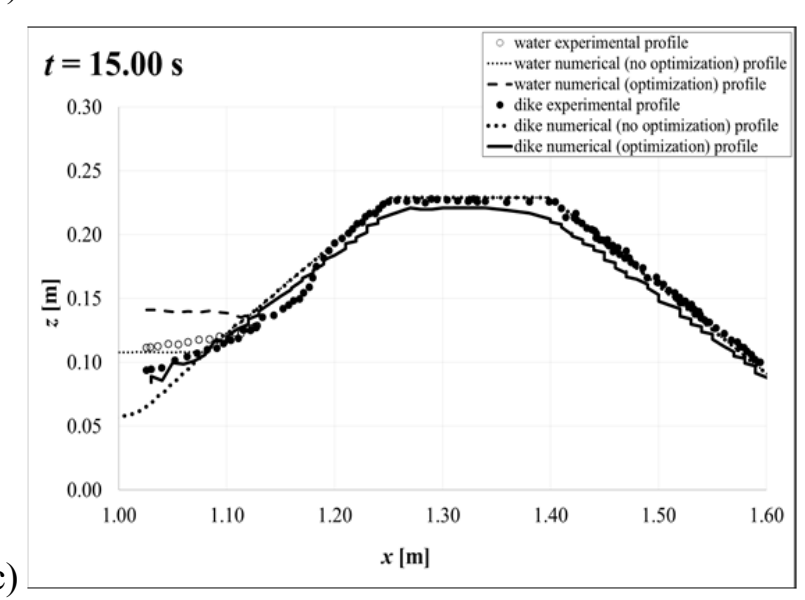

Fig. 7. Test B1: comparison between numerical (with and without optimization) and experimental results in terms of water and dike profiles at times a) 1.40 , b) 3.00 and c) $15.00 \mathrm{~s}$, respectively, after the gate removal.

Table 4. Worst relative errors for sand and water profiles.

\begin{tabular}{ccc|cc}
\hline TEST & $\frac{\left|\delta_{\text {num }, s}-\delta_{\text {exp }, s}\right|}{\delta_{\text {exp }, s}} \cdot 100$ & $\frac{\left|\delta_{\text {num }, \text { optim }, s}-\delta_{\text {exp }, s}\right|}{\delta_{\text {exp }, s}} \cdot 100$ & $\frac{\left|\delta_{\text {num }, w}-\delta_{\text {exp }, w}\right|}{\delta_{\text {exp }, w}} \cdot 100$ & $\frac{\left|\delta_{\text {num }, \text { optim }, w}-\delta_{\text {exp }, w}\right|}{\delta_{\text {exp }, w}} \cdot 100$ \\
\hline A1 & 40.7 & 32.7 & 26.0 & 37.1 \\
A2 & 100.7 & 96.2 & 64.9 & 50.8 \\
A3 & 178.3 & 127.6 & 163.6 & 112.0 \\
B1 & 27.6 & 13.5 & 26.8 & 12.1 \\
B2 & 34.5 & 29.6 & 29.6 & 17.3 \\
B3 & 46.4 & 41.8 & 47.8 & 46.8 \\
\hline
\end{tabular}




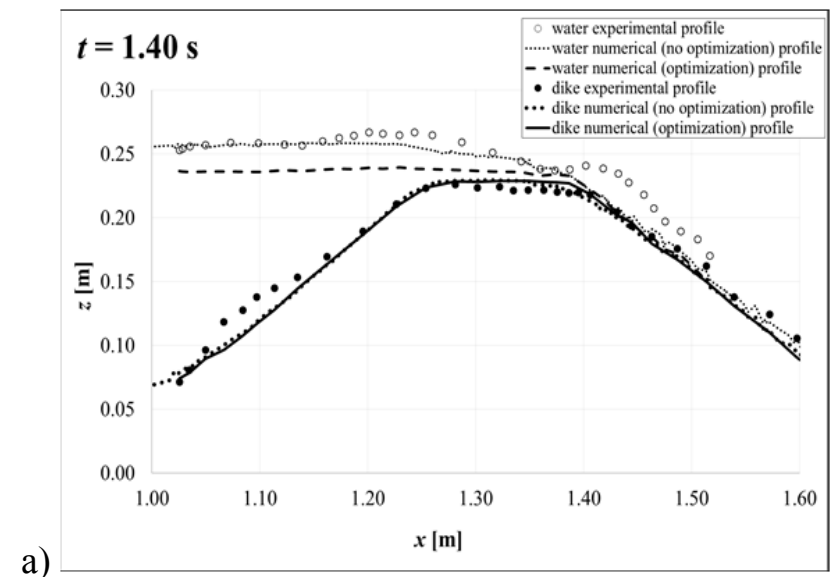

a)

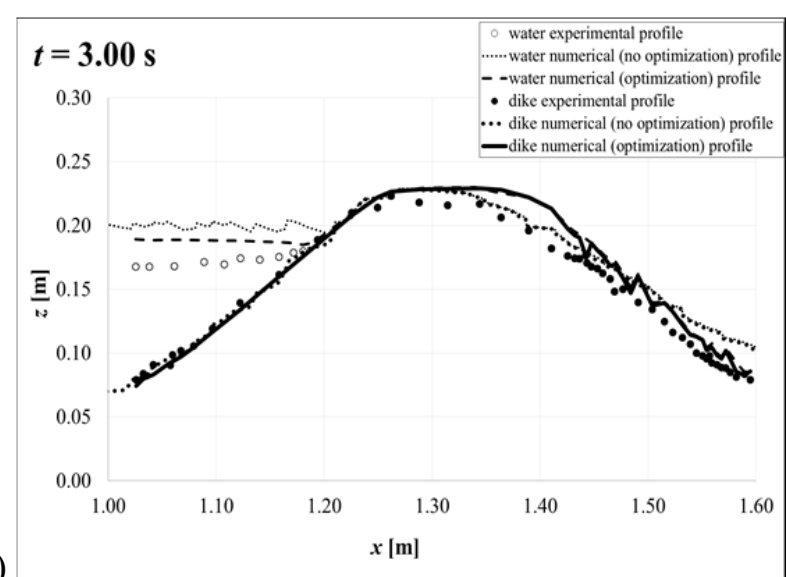

b)

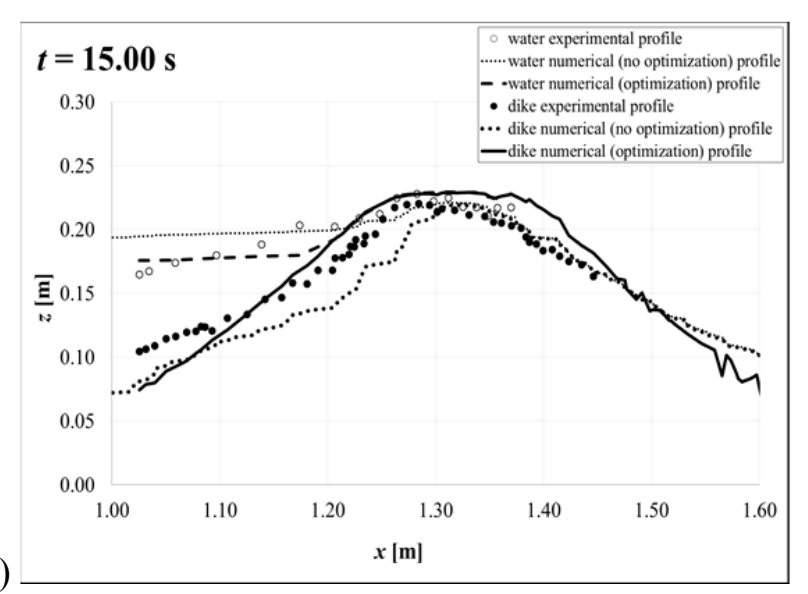

Fig. 8. Test B2: comparison between numerical (with and without optimization) and experimental results in terms of water and dike profiles at times a) 1.40, b) 3.00 and c) $15.00 \mathrm{~s}$, respectively, after the gate removal.

\section{CONCLUSIONS}

Calibration of parameters in the application of most mathematical models of empirical derivation for the simulation of real physical problems is still a tough task in several problems. Nevertheless, the success of the application of the numerical model is strongly dependent on the reliability of its calibration.

The optimum achievement would be the independence of the mathematical models from parameters, but since most of them necessarily contain empirical coefficients, it is still necessary to count on a reliable estimation of them.

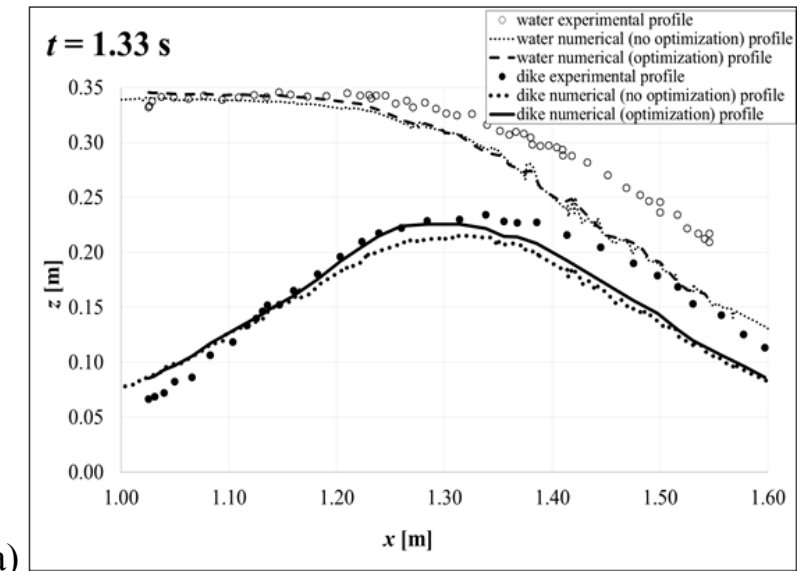

a)

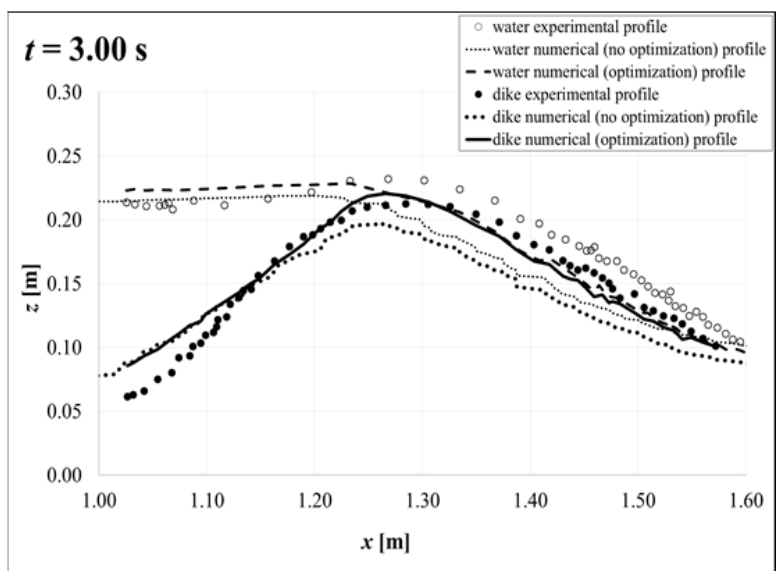

b)

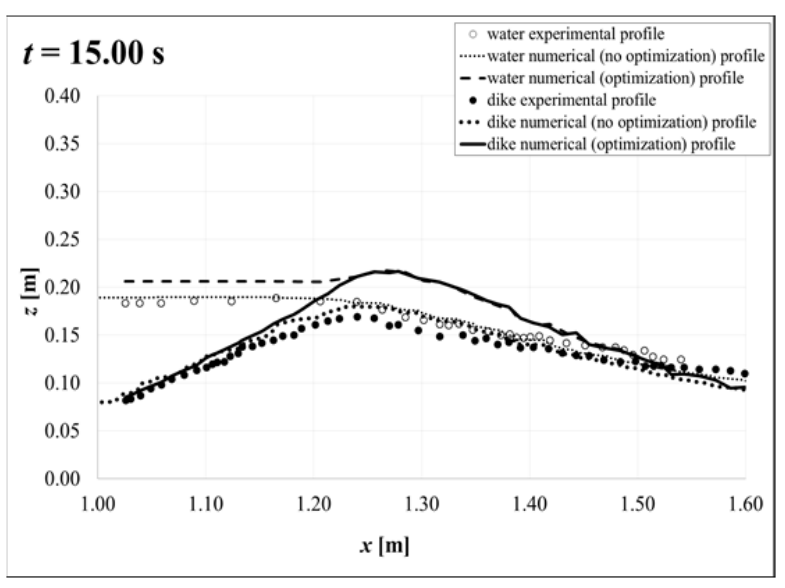

Fig. 9. Test B3: comparison between numerical (with and without optimization) and experimental results in terms of water and dike profiles at times a) 1.40, b) 3.00 and c) $15.00 \mathrm{~s}$, respectively, after the gate removal.

The calibration can be of great complexity, especially for models with a large number of parameters. Engineers and researchers traditionally adjust the parameters through manual trial-and-error procedures and comparison against available experimental data, often varying a single parameter and assuming the other ones as constant. Yet, this approach to calibration is subjective and cumbersome. Since the models themselves are increasingly sophisticated, it seems more proper to look at more advanced calibration procedures.

In this work, in particular, the derivative-free Nelder-Mead algorithm has been adopted to optimize the multi-parameter calibration of a two-phase depth-averaged model (Greco et al., 
2012), already applied in previous works to simulate morphodynamic processes, such as for example dike erosion by overtopping (Evangelista, 2015).

Results show significant advantages of the calibration optimization in terms of calculation times, computational efficiency and objectivity of the results with respect to the previous results obtained through a trial-and-error calibration.

The optimization algorithm here adopted for the multiparameter calibration can be, therefore, supposedly applied with successful results to different mathematical models in river hydraulics and in general in the fields of hydraulics involving geomorphic processes (erosion/deposition of movable bottoms, sediment transport).

\section{REFERENCES}

Capart, H., Young, D., 2002. Two-layer shallow water computations of torrential geomorphic flows. In: Bousmar, D., Zech, Y. (Eds.): Proc. Int. Conf. River Flow 2002. Balkema Rotterdam, Vol. 2, pp. 1003-1012.

Evangelista, S., Altinakar, M.S., Di Cristo, C., Leopardi, A., 2013a. Simulation of dam-break waves on movable beds using a multi-stage centered scheme. Int. J. Sediment Res., 28, 3, 269-284.

Evangelista, S., Greco, M., Iervolino, M., Leopardi, A., Vacca, A., 2013b. A new algorithm for unstructured grids and bankfailure mechanisms in morphodynamic models. In: Proc. XXXV IAHR Intern. Biennial Congress. Tsinghua University Press, Tsinghua, pp. 1-9.

Evangelista, S., de Marinis, G., Di Cristo, C., Leopardi, A., 2014a. Dam-break dry granular flows: Experimental and numerical analysis. WSEAS Trans. Environ. Dev., 10, 382392.

Evangelista, S., Leopardi, A., Mingarelli, M., 2014b. Dike erosion due to a sudden-wave overtopping: Preliminary results. In: Proc. Int. Conf. River Flow 2014. IAHR, Lausanne, Switzerland, pp. 1661-1667.

Evangelista, S., 2015. Experiments and numerical simulations of dike erosion due to a wave impact. Water, 7, 10, 58315848 .

Evangelista, S., Di Cristo, C., Leopardi, A., de Marinis, G., 2015a. Experiments of dike erosion due to a wave impact. In: Proc. 8th WSEAS Int. Conf. on Environmental and Geological Science and Engineering (EG '15). WSEAS Press, pp. 38-44. ISBN: 978-1-61804-314-6.

Evangelista, S., Giovinco, G., Wanik, L., 2015b. Single vs multi parameter calibration for the numerical simulation of submerged flows in jet grouting applications. International Journal of Mechanics, 9, 252-259, ISSN: 1998-4448.

Evangelista, S., Greco, M., Iervolino, M., Leopardi, A., Vacca, A., 2015c. A new algorithm for bank-failure mechanisms in 2d morphodynamic models with unstructured grids. Int. J. Sediment Res., 30, 4, 382-391.
Finley, J.R., Pintér, J.D., Satish, M.G., 1998. Automatic model calibration applying global optimization techniques. Environ. Model. \& Assess., 3, 1, 117-126.

Garegnani, G., Rosatti, G., Bonaventura, L., 2011. Free surface flows over mobile bed: Mathematical analysis and numerical modeling of coupled and decoupled approaches. Communications in Applied and Industrial Mathematics, 2, 1, 1-22.

Greco, M., Iervolino, M., Leopardi, A., 2008. Discussion on divergence form for bed slope source term in shallow water equations. J. Hydraul. Eng., ASCE, 134, 676-678.

Greco, M., Iervolino, M., Leopardi, A., Vacca, A., 2012. A two-phase model for fast geomorphic shallow flows. Int. J. Sediment Res., 27, 409-425.

Harten, A., Lax, P.D., van Leer, B., 1983. On upstream differencing and Godunov-type schemes for hyperbolic conservation laws. SIAM Rev., 25, 35-61.

Kleidorfer, M., Möderl, M., Fach, S., Rauch, W., 2009. Optimization of measurement campaigns for calibration of a conceptual sewer model. Water Sci Technol., 59, 8, 1523-1530.

Li., J., Cao, Z., Pender, G., Liu, Q., 2013. A double layeraveraged model for dam-break flows over mobile bed. J. Hydraul. Res., 51, 5, 518-534.

Lo, D.-C., Wei, C.-C., Tsai, E.-P., 2015. Parameter automatic calibration approach for neural-network-based cyclonic precipitation forecast models. Water, 7, 7, 3963-3977.

Nelder, J.A., Mead, R., 1965. A simplex method for function minimization. The Computer Journal, 7, 4, 308-313.

Rosatti, G., Begnudelli, L., 2013. A closure-independent generalized Roe solver for free-surface, two-phase flows over mobile bed. J. Comput. Phys., 255, 362-383.

Savary, C., Zech, Y., 2007. Boundary conditions in a two-layer geomorphological model: Application to a hydraulic jump over a mobile bed. J. Hydraul. Res., 45, 3, 316-332.

Scilab, 2012. G1 - Scilab Enterprises, Scilab: Free and Open Source software for numerical computation. Version 5.5.2. http://www.scilab.org.

Smith, P.J., Dance, S.L., Baines, M.J., Nichols, N.K., Scott, T.R., 2009. Variational data assimilation for parameter estimation: application to a simple morphodynamic model. Ocean Dynamics, 59, 697-708.

Smith, P.J., Thornhill, G.D., Dance, S.L., Lawless, A.S., Mason, D.C., Nichols, N.K., 2013. Data assimilation for state and parameter estimation: application to morphodynamic modeling. Q. J. R. Meteorol. Soc., 139, 671, 314-327.

Swartenbroekx, C., Soares-Frazão, S., Staquet, R., Zech, Y., 2010. Two-dimensional operator for bank failures induced by water-level rise in dam-break flows. J. Hydraul. Res., 48, $3,302-314$

Received 11 April 2016 Accepted 15 November 2016 